\title{
Numerical solutions of metal plastic flow in compression tests with sinusoidal profile of die roughness
}

\author{
K. Mogielnicki*, J. Piwnik* \\ *Faculty of Mechanical Engineering, Bialystok University of Technology, Bialystok, Poland, \\ E-mail: k.mogielnicki@pb.edu.pl,j.piwnik@pb.edu.pl \\ cross $^{\text {ref }}$ http://dx.doi.org/10.5755/j01.mech.22.6.13574
}

\section{Introduction}

In the case of metal plastic deformation processes, velocities of material particles at the rubbing surfaces have different values at various points of contact. To deform the metal in the presence of friction, it is necessary to use a much greater force than it would from the plastic resistance of the workpiece. With appearing of friction, the force needed to strain and energy consumption increases. The phenomenon of friction is one of the main causes of heterogeneity of strain. Areas of the volume located near the source of the friction are increasingly exposed to the forces impeding the deformation than the further parts from the contact zone.

In the compression as well as in the tensile test, without considering the influence of friction, there is an uniaxial state of stress. Hence, from a theoretical point of view, compression test is a simple inversion of the tensile one. While the states of stress in the tensile and compression tests differ only in signs, the practical conduction of compression one gives rise to a number of difficulties.

Compressed sample must have quite substantial cross section dimensions in relation to its height. Otherwise, there is a danger of buckling under the influence of axial force. The difficulties are also in providing to the compressed sample exactly axial force. Eccentric force leads to undesirable bending of the sample. In the case of plastic materials, compressed between two flat slabs of the press sample, changes its cylindrical shape into a barrel one. The reason for this phenomenon is friction forces occurring at the contact surfaces. In the case of small samples compressing, size effect associated with the roughness of dies appears in addition. That effect manifests itself in stress and strain changes in the flowing sample. Miniaturization of forming processes introduces a need for a more thorough understanding the peculiarities emerging at the contact of toolworkpiece rubbing pair.

The problem in modeling of forming processes in microscale is that the die-workpiece interface effects become very significant. Geiger [1] has shown that the use of the traditional friction coefficient or friction factor can lead to erroneous results. In numerical analysis, it can be included the impact of degree of a tool roughness on the material deformation process and thus the friction factor value. In work [2] the friction model for rough contact of plastic material with rigid tool is shown. It is based on a simplified geometry of the tool top layer, which actual profile reflects a triangular wave. Its length $A R$, height $R$ and angle $\alpha$ are the average surface roughness parameters taken from the roughness profile. These profile parameters are defined by the standard ISO 12085:1999/AC:2009 and can be measured with profilometers. In that way, a geometry of the tool surface layers were modeled in FE simulations of the forward microextrusion processes [3, 4], where a significant effect of containers roughness on the material flow was shown.

In papers $[5,6]$ behavior of the surface layers of the compressed axisymmetric samples is described. Tool roughness was modeled in the form of a rigid triangular wave and the wave of interconnected arcs - model of machined by turning surface in FE simulations. Authors of $[7,8]$ conducted numerical simulation of the ring compression with taken into account dies roughness. Working surface was modeled in the form of a rigid sinusoidal curve with zero friction. The curve was referenced to the parameter $\mathrm{Ra}$ and the friction factor was determined as a $m=f(a, t)$.

This paper contains numerical analysis of influence of the die roughness on the material plastic flow during compression in dry conditions in the microscale - size effect. Roughness was modeled in the form of a sinusoidal curve, which was designated based on the roughness motifs. Simulations of compression between parallel and conical dies were conducted.

\section{Investigation assumptions of numerical modeling}

Motifs method according to ISO 12085:1999/AC:2009 divided roughness profile into so-called motifs. Motif is a part of the original profile between the highest points of two local, not necessarily neighboring, peaks of the profile. This method allows characterizing inequalities using the length and depth of the motif and does not require any filter of the profile. Fig. 1 shows the profile of surface roughness obtained by WEDM. Achieved values of the length and depth of motifs from this profile were used to create the average roughness profile composed of triangular elements (Fig. 2) with the following parameters: $R=4.89 \pm 1.09 \mu \mathrm{m}$ and $A R=16.66 \pm 2.05 \mu \mathrm{m}$.

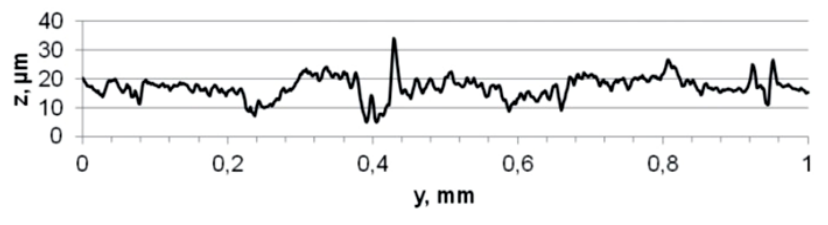

Fig. 1 Surface roughness profile 


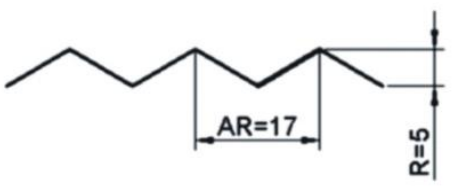

Fig. 2 Roughness motifs, $\mu \mathrm{m}$

Sinusoidal curve described by the formula $z=0.5 R \sin (2 \pi y / A R)$ was imposed on the achieved motifs (Fig. 3). That curve was used to the dies roughness profile modeling in the numerical analysis of the cylinder compression. Introduction of the rigid, sinusoidal tool surface model allows modeling tool roughness, based on experimentally obtained roughness profile.

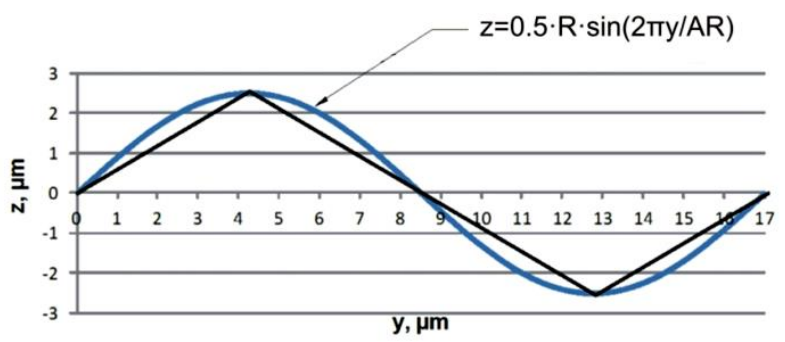

Fig. 3 Imposed on motifs sinusoidal roughness wave

\section{Compression between parallel dies}

Results of the aluminum cylinder compression in parallel dies numerical analysis are presented below. Perfectly rigid-plastic material $\mathrm{Al} .99,0 \mathrm{Cu}$ with isotropic hardening was used. The analysis was conducted using the DEFORM software based on FEM and on the equations of the theory of plasticity. Dies surfaces were modeled in the form of a sinusoidal curve described by the equation $\mathrm{z}=2.5 \sin (2 \pi / 17)$ with friction factor $m=0$ at the interface. Applied finite element mesh was fine enough to take into account the variable geometry of the dies surfaces. The cylinder in diameter $d=1 \mathrm{~mm}$ and in height $h=1.5 d$ was compressed to a half of its height and its face radius was measured. Fig. 4 presents longitudinal half section of the described model.
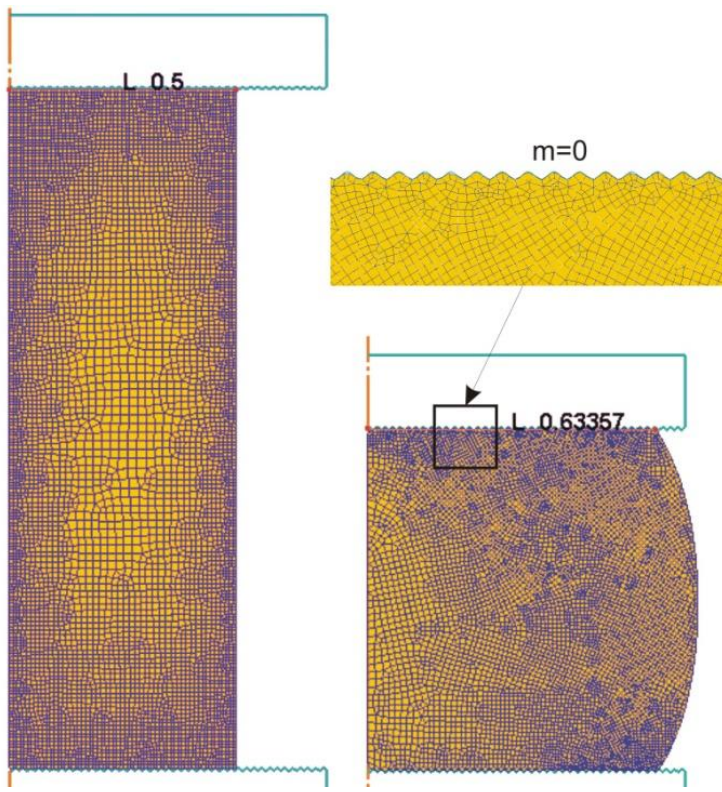

Fig. 4 Numerical model of the cylinder compression between parallel dies with sinusoidal profile
Subsequently 10 similar, conventional simulations of cylinder compression between dies with flat surfaces were performed with successively constant friction factors $\mathrm{m}$; where: $m=\tau_{f} / k, \tau_{f}$ - friction stress, $k$ - shear yield stress. Obtained lengths of compressed cylinders faces radiuses are shown in Fig. 5.

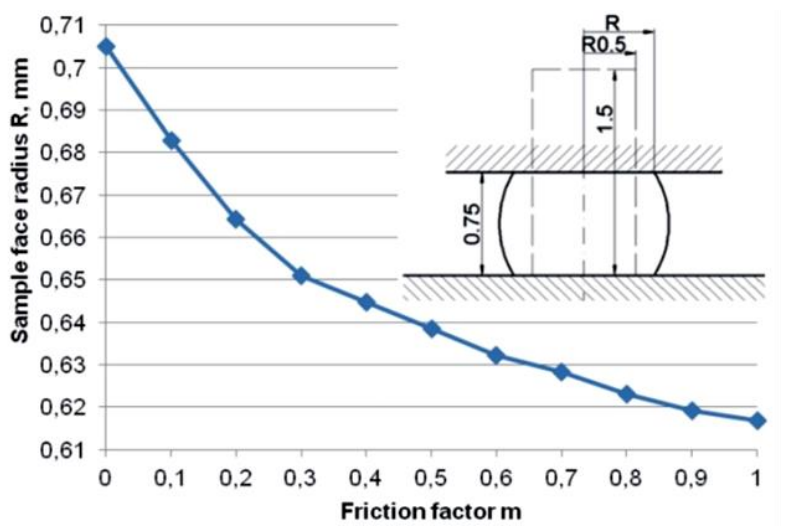

Fig. 5 Radiuses lengths of compressed cylinders faces $m \in\langle 0 ; 1>$

Achieved chart shows, that the length of radius obtained for compression model with sinusoidal profile of dies corresponds to the length of radius for $m=0.6$ with the flat one. Finite element mesh for that model is shown in Fig. 6.

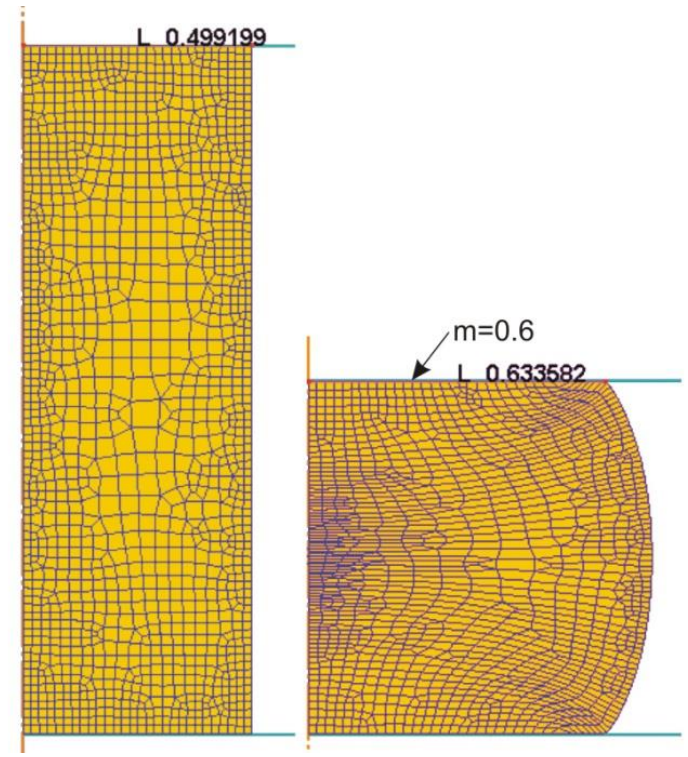

Fig. 6 Numerical model of cylinder compression between parallel dies with flat profile

Stress and strain distributions obtained for both numerical models of the cylinder compression in half sections are presented below. Charts shows values present at the surfaces radiuses. In the model with the sinusoidal profile, measuring line was placed at the valleys level of the roughness wave.

Effective stress (Fig. 7) and strain (Fig. 8) distributions are approximate for both profiles of analyzed dies. However, measurement of these parameters at the radius of the contact zone indicates their growth in the presence of a sinusoidal profile (Fig. 7, a and 8, a). In this case, there is also an increase in the contact area with increased parameters and minor fluctuations in their value. 


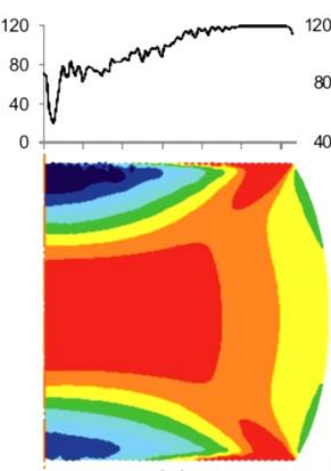

a

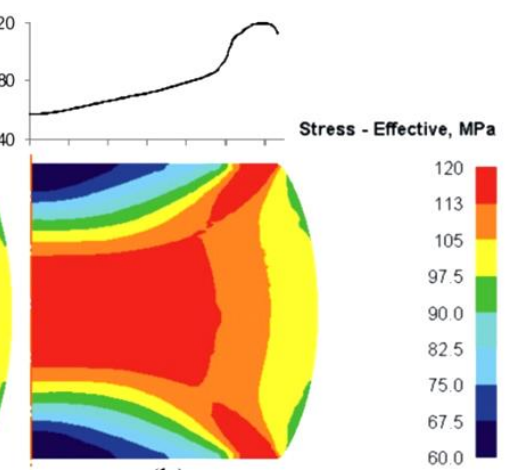

b
Fig. 7 Effective stress distributions in compressed cylinder between parallel dies with the profile: a - sinusoidal; b - flat

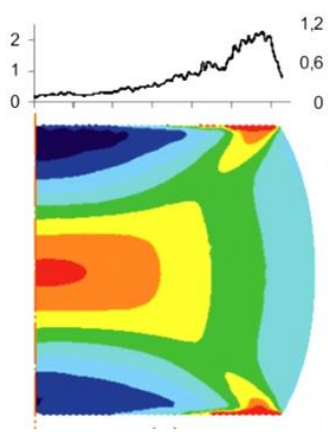

a

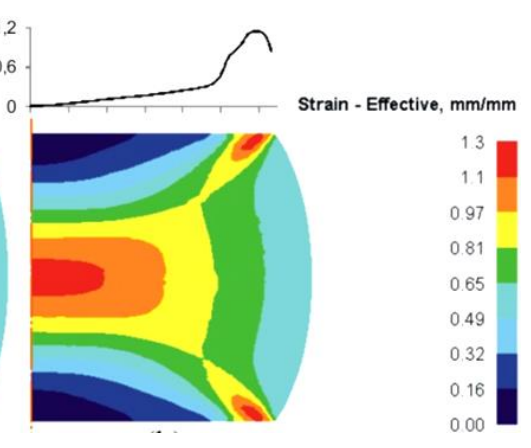

b
Fig. 8 Effective strain distributions in compressed cylinder between parallel dies with the profile: a - sinusoidal; b - flat

Directional stress $R$ (Fig. 9) and $Z$ (Fig. 10) distributions present similar dependings to the case of effective one. Model of compression with sinusoidal profile (Fig. 9, a and 10, a) demonstrates different values of these parameters measured at the upper and lower part. They have a smaller fluctuations frequency in the lower part of the model.

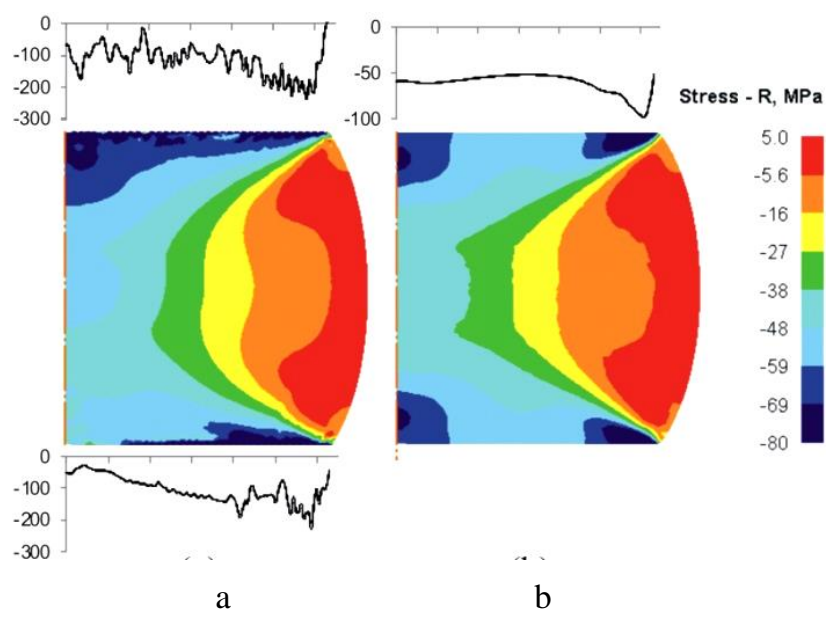

Fig. 9 Stress $R$ distributions in compressed cylinder between parallel dies with the profile: a - sinusoidal; b - flat

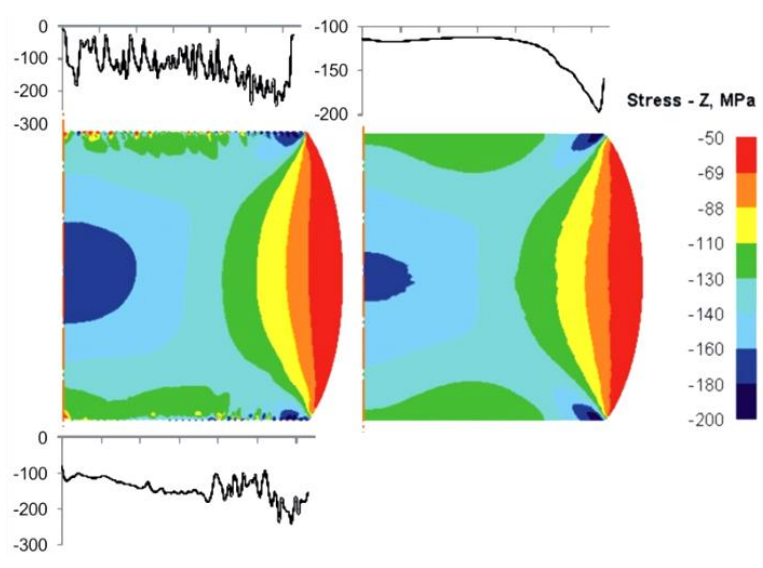

a

b

Fig. 10 Stress $Z$ distributions in compressed cylinder between parallel dies with the profile: a - sinusoidal; b - flat

\section{Compression between conical dies}

In order to determine the angle of a conical die $\alpha$ corresponding to the friction factor $\mathrm{m}$, plastic friction model presented in Fig. 11 was designed. Value of the tangent of the angle was determined from the equation $m=\operatorname{tg} \alpha=\frac{\tau_{f}}{k}<1 \rightarrow \alpha<45^{\circ}$.

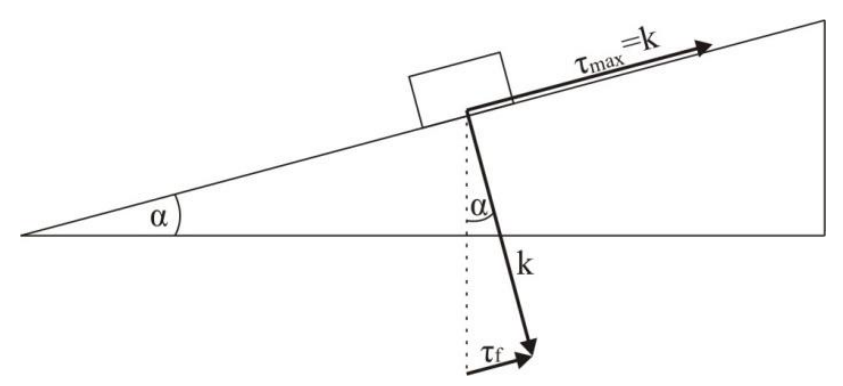

Fig. 11 Angle of the cone forming

For the above designated friction factor $m=0.6$, equivalent to sinusoidal wave $z=2.5 \sin (2 \pi / 17) y$, the angle of cone forming $\alpha=31^{\circ}$ was determined. Using that angle, the shape of the cylindrical surface of cylinder should not turn into a barrel while compressing. Based on above assumptions two numerical models of cylinder compression between conical dies were designed.
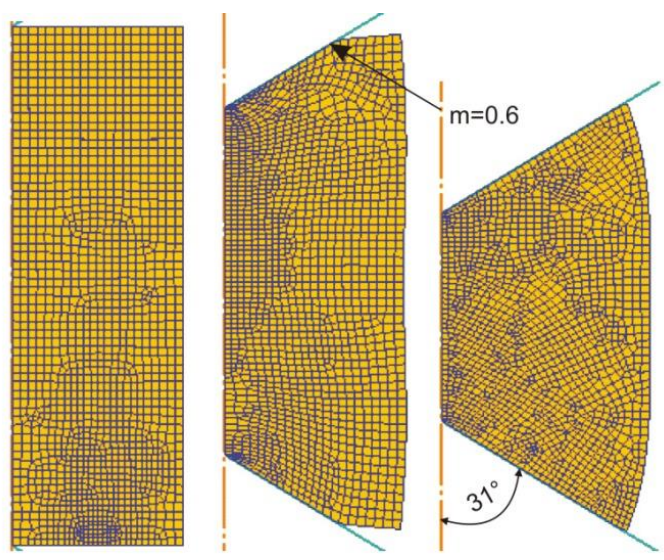

Fig. 12 Flow stages of cylinder model compressed between conical dies with a flat profile 


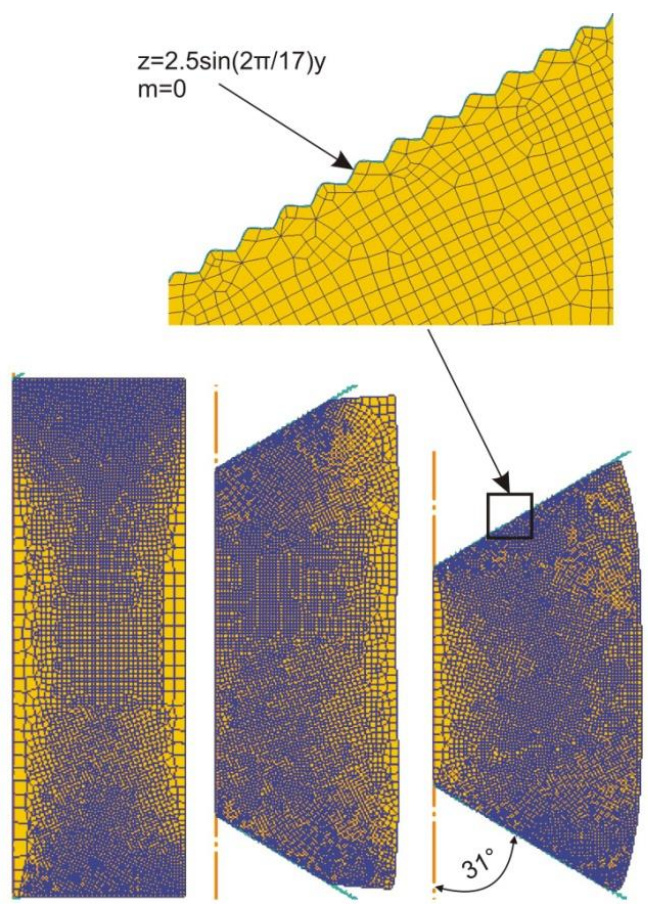

Fig. 13 Flow stages of compressed between conical dies cylinder model with the tool roughness sinusoidal profile

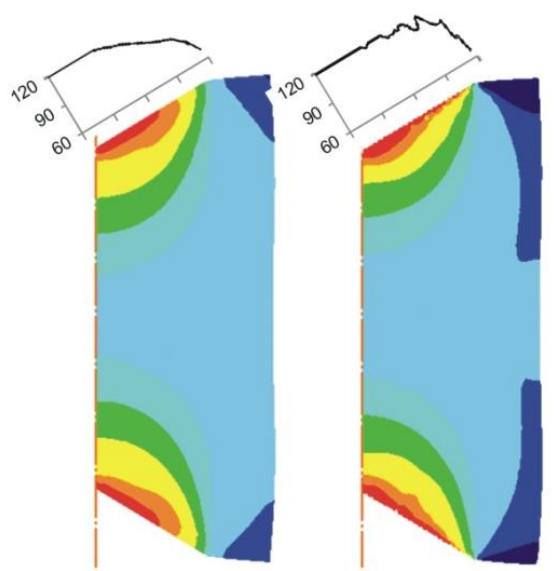

Stress-Effective, MPa

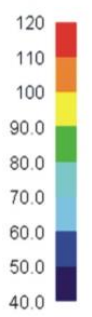

a

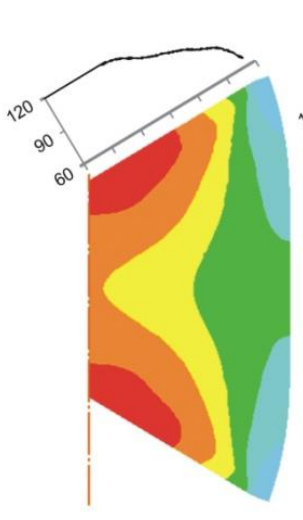

c b

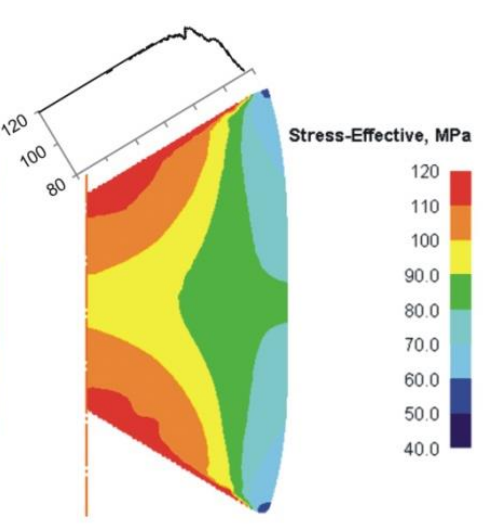

d
Fig. 14 Stress effective distributions in the compressed between conical dies cylinder model with a tool roughness profile: $a, c$ - flat; $b, d$ - sinusoidal

In the first model (Fig. 12) anvils had flat surfaces, with constant friction factor $m=0.6$. In the second one
(Fig. 13), they had a sinusoidal profile and zero friction factor at the tool-sample contact. Dimensions and material of the sample were left as above. In both analyzed cases, the upper and lower parts of the models cylindrical surfaces were curving during advanced stage of compression.

Analysis of effective stress (Fig. 14) and strain (Fig. 15) distributions in the compressed between conical anvils cylinder model revealed, similarly to the case of flat ones, increase in high values in contact area with a presence of sinusoidal profile (Fig. 14, b and d). A similar relationship was found also at the tool-workpiece interface in the case of effective strain distributions (Fig. 15, b and d).

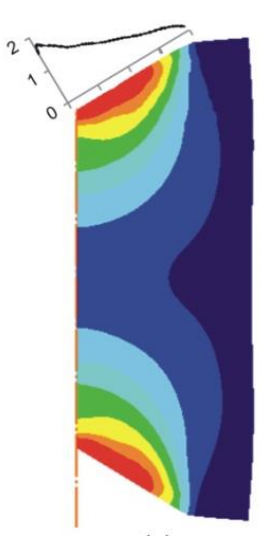

$\mathrm{a}$

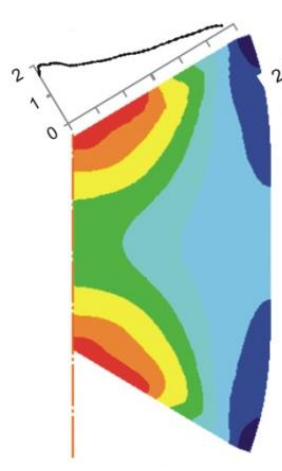

c

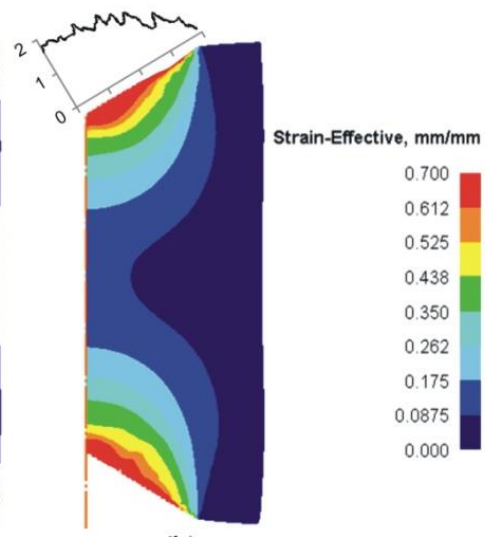

b

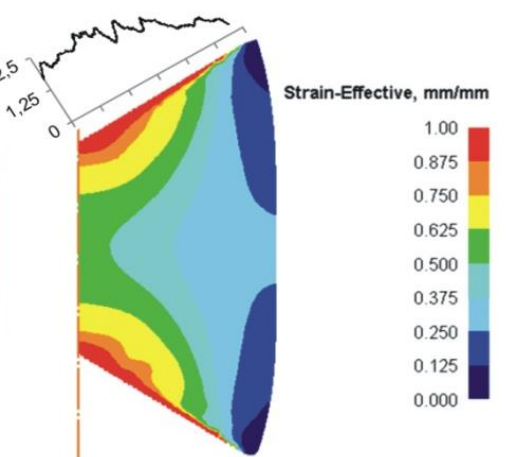

d
Fig. 15 Strain effective in the compressed between conical dies cylinder model with a tool roughness profile: a, c - flat; b, d - sinusoidal

Application of sinusoidal roughness profile of conical dies caused a significant frequency fluctuations in value of the directional stresses $R$ and $Z$ (Fig. 16 and Fig. 17). Stress $R$ at the interface (Fig. 16, b and c) reached locally much higher value than in the case of a flat profile. In turn, values of the stress $Z$ for both anvils profiles, alike during compression (Fig. 17, a and b) and in the final stage of the process (Fig. 17, c and d), run on approximately the same level.

Compression forces obtained for both shapes of anvils differ significantly (Fig. 18). However, application of sinusoidal roughness profiles not affects clearly on achieved forces compared with the flat ones. In the case of parallel dies achieved forces are equal. For the conical ones, application of sinusoidal profile caused a slight reduction of the compression force value. 


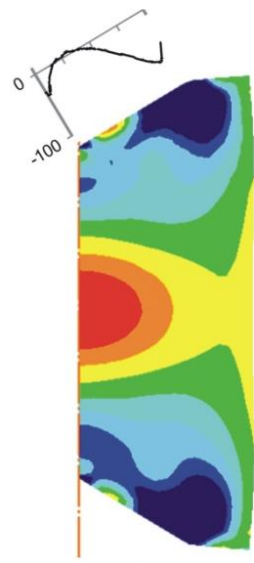

a

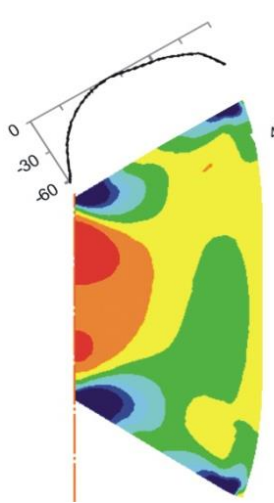

C

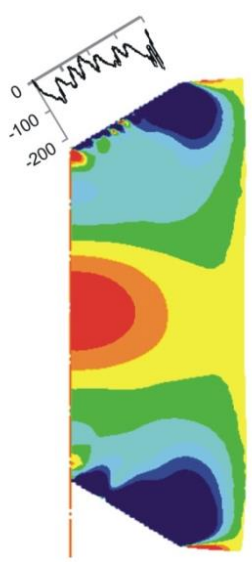

b

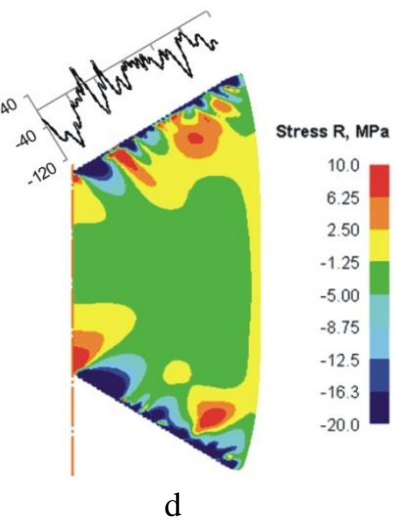

d

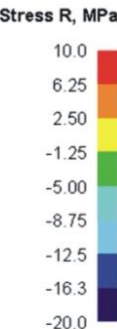

10.0
6.25
2.50
-1.25
-5.00
-8.75
-12.5
-16.3
-20.0

Fig. 16 Stress $\mathrm{R}$ distributions in the compressed between conical dies cylinder model with a tool roughness profile: a, c - flat; b, d - sinusoidal

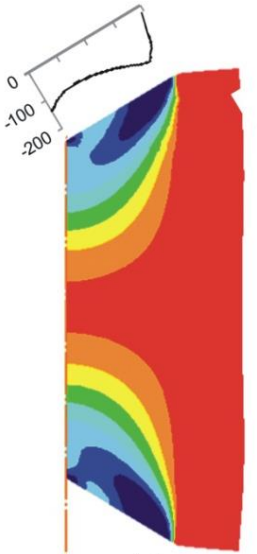

a

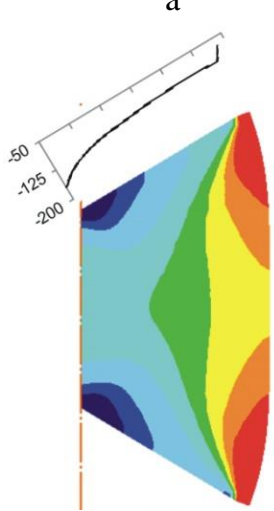

c

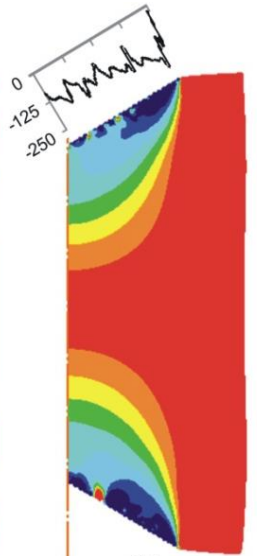

b

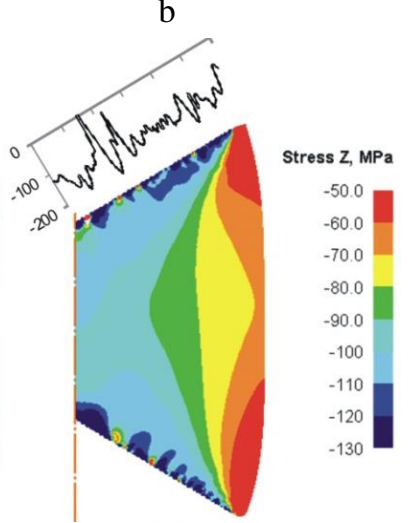

d
Fig. 17 Stress $\mathrm{Z}$ distributions in the compressed between conical dies cylinder model with a tool roughness profile: a, c - flat; b, d - sinusoidal

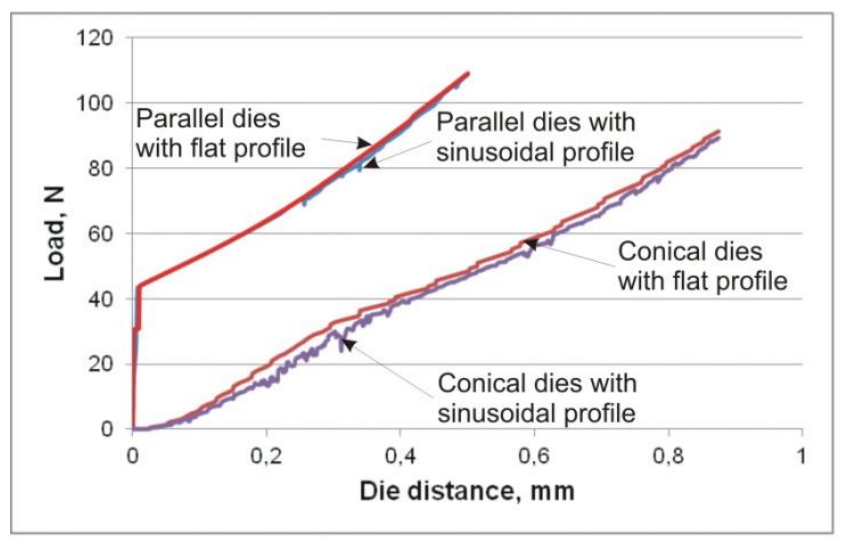

Fig. 18 Compression forces

\section{Conclusions}

Paper contains results of numerical analysis of cylinder compression between parallel and conical dies, with taking into account the tool surface roughness microgeometry. The roughness was modeled in the form of sinusoidal curve, imposed on a triangular motifs wave, obtained on the basis of roughness profile. Zero friction factor at the toolworkpiece interface has been given. Obtained results were compared with analogous, conventional models of compression test with flat dies surfaces and a constant friction factor at the interface. In the case of parallel dies, the friction factor value was designated by comparing lengths of face radiuses of compressed samples. The cone forming angle of conical dies was established on the basis of the created plastic friction model. In the course of investigations it has been shown, that use of sinusoidal roughness profile causes increase in stress and strain in material layers flowing in its vicinity. There was no influence of sinusoidal profile presence on the compression forces in both analyzed tests. This confirms the legitimacy of its use in numerical models of plastic deformation of metals. Presented at work method of tool roughness describing using a sinusoidal wave, allows revealing changes in the deformation process of metals in microscale - size effect, induced by heightened influence of a tool roughness.

\section{References}

1. Geiger, M.; Kleiner, M.; Eckstein, R.; Tiesler, N.; Engel, U. 2001. Microforming, Annals of CIRP 50(2): 445-462.

http://dx.doi.org/10.1016/S0007-8506(07)62991-6.

2. Challen, J.M.; Oxley, P.L.B. 1979. An explanation of the different regimes of friction and wear using asperity deformation models, Wear 53: 229-243.

http://dx.doi.org/10.1016/0043-1648(79)90080-2.

3. Piwnik, J.; Mogielnicki, K. 2010. The friction influence on stress in micro extrusion, Archives of Foundry Engineering 10:451-454. Available from Internet: http://www.afe.polsl.pl/index.php/pl/791/the-frictioninfluence-on-stress-in-micro-extrusion.pdf.

4. Piwnik, J.; Mogielnicki, K. 2014. Experimental and FE analysis of aluminium alloy plastic flow in the forward micro-extrusion processes, Archives of Metallurgy and Materials 59(2): 521-525. http://dx.doi.org/10.2478/amm-2014-0086. 
5. Vidal-Sallé, E.; Boutabba, S.; Cui, Y.; Boyer, J.C. 2008. An improved "plastic wave" friction model for rough contact in axisymmetric modeling of bulk forming processes, 11th ESAFORM Conference on material Forming, Lyon. http://dx.doi.org/10.1007/s12289-008-0132-y.

6. Vidal-Sallé, E.; Maisonnette-Masson, S., Boyer, J.C. 2009. About the validity of the plastic wave model for an actual roughness of axisymmetric tooling in bulk forming, International Journal of Material Forming 2: 217-220.

http://dx.doi.org/10.1007/s12289-009-0447-3.

7. Jeon, H.J.; Bramley, A.N. 2007. A friction model for microforming, The International Journal of Advanced Manufacturing Technology 33: 125-129.

http://dx.doi.org/10.1007/s00170-006-0608-1.

8. Becker, P.; Jeon, H.J.; Chang, C.C.; Bramley, A.N. 2003. A geometric approach to modelling friction in metal forming, CIRP Annals-Manufacturing Technology 52(1): 209-212.

http://dx.doi.org/10.1016/S0007-8506(07)60567-8.
K. Mogielnicki, J. Piwnik

NUMERICAL SOLUTIONS OF METAL PLASTIC FLOW IN COMPRESSION TESTS WITH SINUSOIDAL PROFILE OF DIE ROUGHNESS

S u m m a r y

During metal bulk-forming in microscale the socalled size effect, making changes in the material deformation process should be taken into account. One of the factors having a significant influence on the flow mechanics is a die roughness. Paper contains results of numerical analysis of the cylindrical sample compression between anvils with sinusoidal profile of roughness and with zero friction factor at the tool-workpiece interface in dry conditions. Two models of dies were used: parallel and conical one. Obtained data were compared to analogous, conventional models of compression tests with flat dies and with constant friction factor - equivalent to sinusoidal wave. Sinusoidal curve modeling the die roughness was determined from the surface roughness profile and using the motifs method. Presented analysis revealed increase in value and in variability of stress and strain in layers flowing in the vicinity of the wave - size effect. No impact of the wave presence on the compression forces values was obtained.

Keywords: Compression test, FEM, microforming, size effect, rough friction. 